\title{
Diffractive digital lensless holographic microscopy with fine spectral tuning
}

\author{
Omel Mendoza-Yero, ${ }^{1, *}$ Enrique Tajahuerce, ${ }^{1}$ Jesús Lancis, ${ }^{1}$ and Jorge Garcia-Sucerquia ${ }^{2,3}$ \\ ${ }^{1}$ Institut de Noves Tecnologies de la Imatge (INIT), Universitat Jaume I, E 12080 Castelló, Spain \\ ${ }^{2}$ Universidad Nacional de Colombia-Sede Medellin, School of Physics, A.A: 3840-Medellin 050034, Colombia \\ ${ }^{3}$ Departamento de Óptica, Universitat de València, 46100 Burjassot, Spain \\ *Corresponding author: omendoza@fca.uji.es
}

Received April 26, 2013; accepted May 15, 2013;

posted May 20, 2013 (Doc. ID 189619); published June 11, 2013

\begin{abstract}
We experimentally demonstrate an all-diffractive optical setup for digital lensless holographic microscopy with easy wavelength line selection and micrometric resolution. In the proposed system, an ultrashort laser pulse is focused with a diffractive lens (DL) onto a pinhole of diameter close to its central wavelength to achieve a highly spatially coherent illumination cone as well as a spectral line with narrow width. To scan the complete spectrum of the light source the DL is displaced with respect to the pinhole plane. The proposed microscopy setup allows us to spectrally separate contributions from different sections of a sample, which may be attractive for several applications in life sciences. (c) 2013 Optical Society of America

OCIS codes: (050.1940) Diffraction; (090.1995) Digital holography; (110.0180) Microscopy.

http://dx.doi.org/10.1364/OL.38.002107
\end{abstract}

Recent advances in digital holographic microscopy show that 3D images of static or moving objects with micrometer resolution are possible by using only a CCD- or CMOS-based camera within a simply in-line lensless optical setup. The technique known as digital lensless holographic microscopy (DLHM) [1] combines both in-line lensless geometry proposed by Gabor in the late 1940s [2] and digital holography approaches for data processing [3]. Basically, in DLHM the light scattered from the object/sample interferes with the reference incident light to generate a holographic pattern at the camera plane. Then, the recorded intensity is numerically processed, and the object wavefront is reconstructed. By using digital in-line lensless holographic microscopy technique, several applications have been demonstrated, which include but are not limited to showing in situ organisms and their motion in plankton with micrometer resolution [4], investigating microbial life forms in the Canadian High Arctic [5], tracking micrometer sized particles with high NA [6] , analyzing transparent phase objects under femtosecond illumination [7], and successfully imaging latex microspheres, optical fiber, and cancer cells with light sources of varying spatial coherence [8].

In this context, multispectral imaging has become a tool of increasing use, i.e., as a way to discriminate among several components (e.g., proteins or genes) or functions within the cell [9], or to enlarge the range of application of phase-based digital holographic techniques [10]. In DLHM multispectral illumination has been achieved not only with coherent sources like a tunable He-Ne laser [11] or a set of lasers that emit at different wavelength lines [12], but also with incoherent light-emitting diodes [8]. However, the above implementations allow only a discrete set of wavelength lines with full width at half-maximum (FWHM), typically on the order of some tens of nanometers. In contrast, it is well known that the ability to separate spectral contributions from multiple components of a sample depends largely on the number of images collected at different wavelength bands as well as on the width of these wavelength bands. In terms of the spectral image cube or lambda stack, it means that the finer the spectral slicing of the available broadband spectral source the better.

In this Letter, we show an extremely compact and simple optical setup for all-diffractive multispectral DLHM with complete spectral tuning over the whole spectrum of the light source. The optical setup is based on the substitution of the microscopy objective given in most typical in-line DLHM geometries $[1,12]$ by a kinoform DL. Here, it should be pointed out that a DL can be regarded as an optical element that focuses the light by diffraction. Its focal length $F(\lambda)$ varies inversely with the wavelength of the incident light as $F(\lambda)=F_{0} \lambda_{0} / \lambda$, where $F_{0}$ is the main focal length for the wavelength $\lambda_{0}$. In our proposal, the pinhole that is placed at a given focal plane of the DL has a dual role. On one hand, it acts as a spatial filter of the light, removing higherorder aberrations and phase-front distortions, thus providing a secondary illuminating spherical wave. On the other hand, moving the DL with respect to the pinhole plane allows us to select very narrow wavelength bands within the spectrum of the incident light. The combination of a DL (or a Fresnel zone plate) with a small pinhole can be thought as a linear spectrometer whose resolving power $R$, for our experimental conditions (pinhole diameter not larger than the diameter of the Airy disc), can be estimated by the expression $R=D^{2} /\left(8 F_{0} \lambda_{0}\right)$, where $D$ is the diameter of the DL $[\underline{13}, \underline{14}]$.

The proposed optical setup has several desirable features for multispectral DLHM. The present optical setup allows for recording quasi-monochromatic digital holograms even when using broadband sources (i.e., high-pressure xenon-mercury lamps, broadband lightemitting diodes, or ultrashort pulsed radiation). The inherent diffractive nature of our system makes it suitable for applications in the extreme ultraviolet (XUV) or x-ray spectral region, where microscopy objectives cannot be used owing to the absorption of materials. In addition, 
the small thickness of the DLs reduces considerably the dimension of the microscopy device.

In Fig. 1 a schematic diagram of the optical setup is shown. For the experiment a Ti-Sa femtosecond laser that emits pulses of about 12 fs intensity FWHM at a $75 \mathrm{MHz}$ repetition rate, centered at $\lambda_{0}=800 \mathrm{~nm}$ with a $100 \mathrm{~nm}$ FWHM spectral bandwidth is the light source.

The light is focused with a kinoform DL down onto a pinhole with a diameter $d=1 \mu \mathrm{m}$ slightly larger than the largest wavelength, which is $0.88 \mu \mathrm{m}$ in this experiment. The DL has a diameter $D=30 \mathrm{~mm}$, whereas its main focal length is $F_{0}=50 \mathrm{~mm}$ for $\lambda_{0}=800 \mathrm{~nm}$. To cover the whole working area of the DL, the ultrashort pulse was previously expanded by means of a $16 \times$ all-mirror beam expander. In addition, suited neutral filters are used to attenuate conveniently the laser radiation.

The spherical wavefront illuminates the sample that is placed at a fixed distance $z$ from the pinhole. On the surface of a digital screen (CCD camera, model Balser A120F) located at a distance $L=15 \mathrm{~mm}$ from the pinhole, the waves scattered by the sample are superimposed with the portions of the spherical waves that travel with no perturbation. The intensity with no sample is pixelwise subtracted from the intensity of the in-line hologram. Then, the obtained intensity is transferred to a computer for further processing. Owing to the high transmittance of the samples used for DLHM, the intensity of the scattered wave can be neglected. Furthermore, as spherical illumination is utilized, the presence of the twin images does not introduce any nuisance on the reconstructed images [1]. The reconstruction of the holograms is numerically carried out with the help of the Fresnel-Kirchhoff diffraction integral. Details of this process can be found elsewhere, see for instance [15].

In order to show the potential of our microscopy setup to gather high-quality images of the sample at different wavelengths, we filtered different spectral lines of the pulse spectrum. To do that, the DL was axially moved with respect to the pinhole position with a micrometer screw gauge $(R \cong 2800)$. At this point, the CCD camera was temporarily substituted by a commercial spectrometer device to record the spectral lines corresponding to

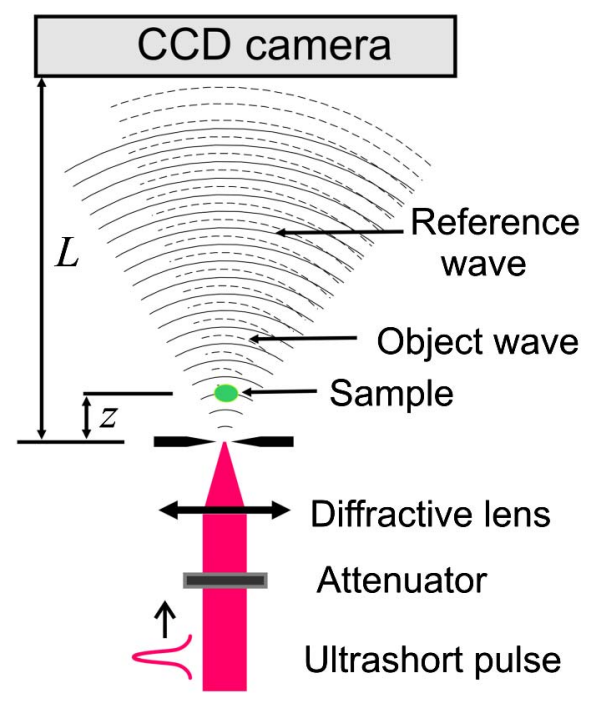

Fig. 1. Schematic of the diffractive DLHM. each position of the DL. In particular, eight spectral lines stepped $20 \mathrm{~nm}$ from 740 to $860 \mathrm{~nm}$ were obtained. Once the positions of the DL were determined, the CCD camera was restored into the optical setup to record the intensity of each spherical wave without a sample. Finally, the sample was introduced and fixed into the optical setup (at the distance $z=5.8 \mathrm{~mm}$ from the pinhole plane). In these conditions, after moving back the DL to the above-determined positions, the corresponding holograms were recorded.

In this work, a paraffin wax section of the head of a fruit fly (Drosophila melanogaster) was used as a sample. The section of the head is about $800 \mu \mathrm{m}$ wide and $10 \mu \mathrm{m}$ in thickness. In Fig. 2, an image of the reconstructed hologram for the spectral line $780 \mathrm{~nm}$ is shown.

From Fig. 2 the complex structure of the fruit fly head can be clearly seen. Some structures of the head with dimensions on the order of a few micrometers (e.g., ommatidia), or even less than one micrometer (e.g., cornea spikes) are highlighted in the top-left inset of Fig. 2. This inset is obtained by $2 \times$ magnifying the encircled region of the image of the section of the head. In the bottom-left part of Fig. 2, the profile of the pulse spectrum together with the selected spectral line of $6 \mathrm{~nm}$ FWHM are also given as an inset.

On the other hand, having good quality images of a sample at different wavelengths could be useful, e.g., to obtain further information on certain optical properties (e.g., reflectance/absorbance of specific regions of the sample). With the proposed microscope, one can spectrally scan the sample with a thin spectral line to get a large number of high-quality images, see for instance Fig. 2. Hence, the possibility of obtaining the so-called lambda stack with several applications in multispectral imaging is apparent [9]. Please note that the hologram reconstruction process guarantees the size of the image coordinates at the reconstruction plane to be independent of the wavelength [12]. This is

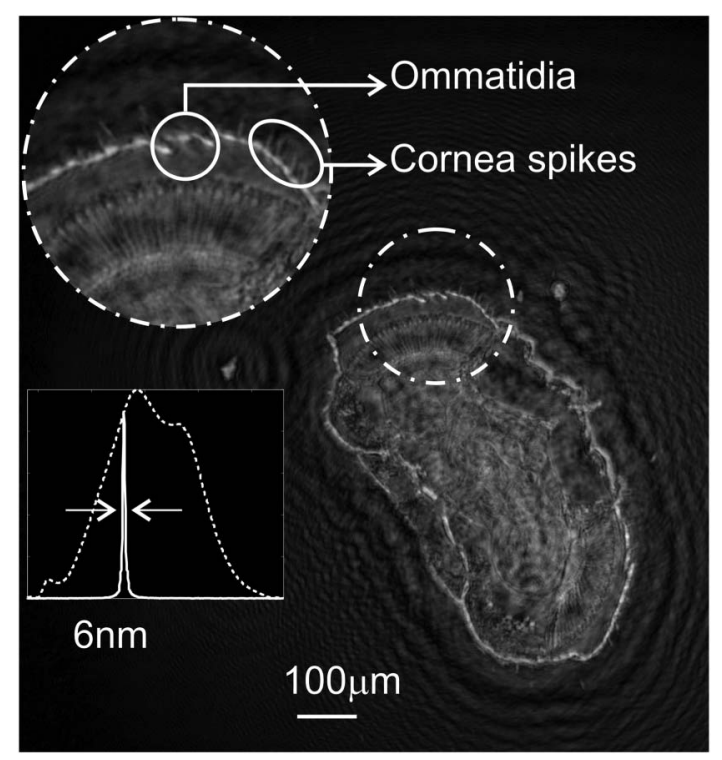

Fig. 2. Reconstructed hologram of a section of the head of a fruit fly for the spectral line centered at $780 \mathrm{~nm}$. Top left: details of the head structure. Bottom left: profile of the pulse spectrum and the selected spectral line. 


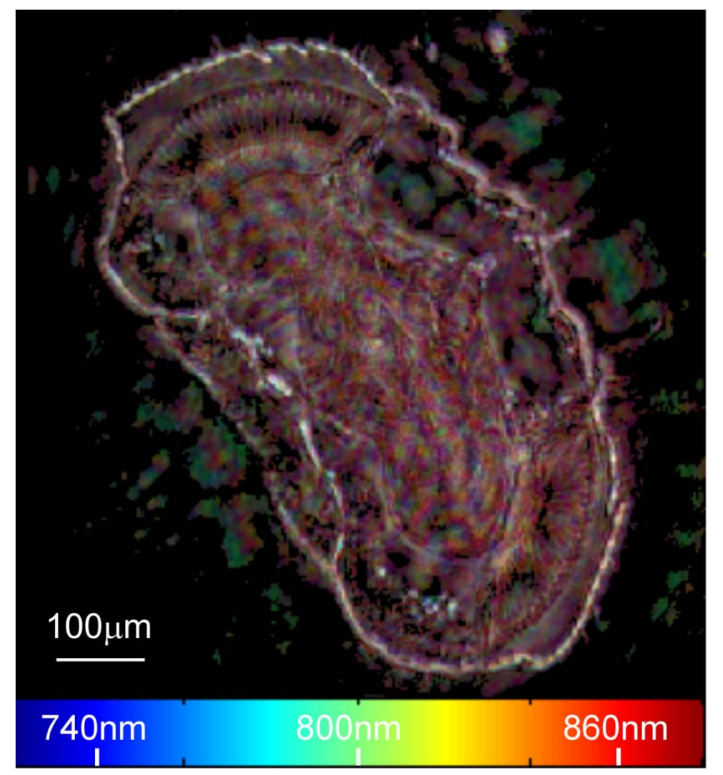

Fig. 3. Pseudo-colored RGB image of a section of the head of the fruit fly obtained from the merging of images corresponding to the spectral lines at 860,800 , and $740 \mathrm{~nm}$.

mandatory to keep the same scale of images for the different wavelengths at the reconstruction plane.

To see the spectral response of our sample to multiwavelength illumination, we merged three images corresponding to the spectral lines at 860,800 , and $740 \mathrm{~nm}$. From this fusing, a pseudo-colored RGB image was generated by associating the $860 \mathrm{~nm}$ image with the red channel, the $800 \mathrm{~nm}$ image with the green channel, and the $740 \mathrm{~nm}$ image with the blue channel. In Fig. $\underline{3}$, the resulting image is shown.

The spectral lines from the pulse spectrum that were used to illuminate the sample are included within the color bar of Fig. 3. After a visual inspection of Fig. $\underline{3}$, one can see that most regions of the head of the fruit fly are wavelength dependent, with a strong preference for the $860 \mathrm{~nm}$ wavelength, whereas the white-colored ommatidia at the border of the section of the head show an even response.

We believe that the power and simplicity of the proposed in-line lensless microscope might be suited for operating within several spectral ranges of the electromagnetic spectrum, at micrometer spatial resolution. More compact and dynamic versions of it can be implemented thanks to the use of, i.e., photolithography techniques and/or spatial light modulators.

This research was funded by the Spanish Ministerio de Ciencia e Innovación and the Generalitat Valenciana through Consolider Programme (SAUUL CSD200700013), and Prometeo Excellence Programme (PROMETEO/2012/021). The authors are also very grateful to the SCIC of the Universitat Jaume I for the use of the femtosecond laser. J. Garcia-Sucerquia gratefully acknowledges the Visiting Scholar Fellowship from the Universidad de Valencia, Colciencias Grant No. 110205024, and UNAL Grant Nos. 110201003 and 110201004.

\section{References}

1. J. Garcia-Sucerquia, W. Xu, S. K. Jericho, P. Klages, M. H. Jericho, and H. J. Kreuzer, Appl. Opt. 45, 836 (2006).

2. D. Gabor, Nature 161, 777 (1948).

3. J. W. Goodman and R. W. Lawrence, Appl. Phys. Lett. 11, 77 (1967).

4. S. K. Jericho, J. Garcia-Sucerquia, W. Xu, M. H. Jericho, and H. J. Kreuzer, Rev. Sci. Instrum. 77, 043706 (2006).

5. S. K. Jerico, P. Klages, J. Nadeau, E. M. Dumas, M. H. Jerico, and H. J. Kreuzer, Planet. Space Sci. 58, 701 (2010).

6. J. F. Restrepo and J. Garcia-Sucerquia, Opt. Lett. 37, 752 (2012).

7. M. Brunel, H. Shen, S. Coetmellec, D. Lebrum, and K. A. Ameur, Appl. Phys. B 106, 583 (2012).

8. J. P. Ryle, S. McDonnell, and J. T. Sheridan, J. Biomed. Opt. 16, 126004 (2011).

9. M. E. Dickinson, G. Bearman, S. Tille, R. Lansford, and S. E. Fraser, Bioimaging 31, 1272 (2001).

10. M. K. Kim, Opt. Lett. 24, 1693 (1999).

11. J. P. Rylea, K. M. Molonyd, S. McDonnelle, T. J. Naughtond, and J. T. Sheridan, Proc. SPIE 7442, 744206 (2009).

12. J. Garcia-Sucerquia, Opt. Lett. 37, 1724 (2012).

13. G. Mínguez-Vega, O. Mendoza-Yero, E. Tajahuerce, J. Lancis, and P. Andrés, IEEE Photon. Technol. Lett. 21, 347 (2009).

14. N. Kitaura, S. Ogata, and Y. Mori, Opt. Eng. 34, 584 (1995).

15. M. H. Jericho and H. J. Kreuzer, in Coherent Light Microscopy, P. Ferraro, A. Wax, and Z. Zalevvsky, eds. (Springer, 2011), pp. 3-30. 